\title{
La lealtad de los colaboradores en una institución educativa privada de nivel superior caso de estudio Celaya, Guanajuato, México
}

Godínez López, Roberto; Luna Correa, José Enrique; Macías Salinas, Silvia

La lealtad de los colaboradores en una institución educativa privada de nivel superior caso de estudio Celaya, Guanajuato, México

Revista Educación, vol. 43, núm. 2, 2019

Universidad de Costa Rica, Costa Rica

Disponible en: http://www.redalyc.org/articulo.oa?id=44058158023

DOI: https://doi.org/10.15517/revedu.v43i2.29246

Esta obra está bajo una Licencia Creative Commons Atribución-NoComercial-SinDerivar 3.0 Internacional. 


\title{
La lealtad de los colaboradores en una institución educativa privada de nivel superior
} caso de estudio Celaya, Guanajuato, México

\author{
Loyalty among Collaborators at a Private Higher Education Institution: A Case Study in Celaya, Guanajuato, \\ México
}

Roberto Godinez López

Universidad de Guanajuato, México

roberto.godinez@gmail.com

http://orcid.org/0000-0003-2612-8996

JoséEnrique Luna Correa
Universidad de Guanajuato, México
enrique_luncor@hotmail.com

Silvia Macias Salinas

Universidad del Centro del Bajio, México

silvia.macias@uniceba.edu.mx
DOI: https://doi.org/10.15517/revedu.v43i2.29246

Redalyc: http://www.redalyc.org/articulo.oa?id=44058158023

Recepción: 21 Junio 2017

Aprobación: 26 Mayo 2019

\section{Resumen:}

La presente investigación analiza el fenómeno de la lealtad de los colaboradores en una institución de educación superior de la ciudad de Celaya, para lo cual se revisa literatura relacionada con estudios previos relacionados con el tema de estudio destacando lo señalado por Flores (2009), en su investigación titulada La lealtad en la cultura del trabajador mexicano de la Ciudad de México, del cual se recuperan las variables de investigación. Este fenómeno se abordó desde un enfoque cuantitativo, estableciendo un alcance correlacional, utilizando un diseño no experimental del tipo transversal, además se estableció como hipótesis de investigación que las variables cualitativas descritas como recursos, trabajo en equipo, jefe directo, gestión de la alta dirección, y retribuciones y compensaciones influyen en la satisfacción y lealtad. Se utilizó un instrumento documental para medir las variables señaladas, empleando una muestra de 76 sujetos de investigación colaboradores de la institución con un nivel de confianza del $95 \%$ y un error del 3\%. Finalmente se analizaron los resultados con la prueba estadística de correlación de Tau-b de Kendall para probar la hipótesis de investigación, encontrándose como principal hallazgo que las variables que mayor influencia significativa sobre la lealtad son trabajo en equipo y retribuciones y compensaciones, lo anterior ofrece evidencia empírica que puede ayudar a la institución en la toma de decisiones para incrementar la lealtad entre sus colaboradores, se recomienda que controlando y estimulando las variables de trabajo en equipo y retribuciones y compensaciones ayudará sustancialmente al incremento de la lealtad de los colaboradores, además estos resultados contribuyen como base de conocimiento para que otras instituciones puedan replicar el método de investigación para que los resultados encontrados también les ayuden en la toma de decisiones acerca de los valores deseados de la lealtad de sus colaboradores.

Palabras clave: Lealtad, Compromiso, Talento Humano.

\section{Abstract:}

This study analyzes the phenomenon of collaborator loyalty at a high school in the city of Celaya. A literature review of previous related studies was first conducted, emphasizing Flores (2009) whose study, Loyalty within the Culture of Mexican Workers in Mexico City, is used as a basis for this study. This phenomenon was addressed from a quantitative perspective, a correlational scope using a non, cross-cutting type experimental design. The hypothesis was that qualitative variables described as resources, team work, direct supervisor, senior administration and compensation influence satisfaction and loyalty. A document was used to measure the aforementioned variables, using a sample of 76 subjects who are also collaborators at the institution with a confidence interval of $95 \%$ and a margin of error of $3 \%$. Lastly, the results were analyzed with the Kendall Tau-B correlational coefficient in order to prove the research hypothesis. The main finding was that the most significant variables that impact loyalty are teamwork, remuneration and compensation, which is empirical proof that can help the institution make decisions to increase loyalty from their collaborators. Controlling and stimulating teamwork, remuneration and compensations variables may significantly help 
increase collaborator loyalty. In addition, those results can be used as a baseline for other institutions to replicate the research models. In this manner, the results obtained can also help the decision-making process about desired collaborator loyalty values.

KEYWORDs: Loyalty, Commitment, Human Talent.

\section{INTRODUCCIÓN}

Todas las empresas cuentan con un objetivo predeterminado por su alcance de producción o de prestación de servicios. Para que este objetivo se cumpla necesita de los trabajadores independientemente del capital financiero y de recursos materiales. La empresa requiere entender la naturaleza de su relación con los trabajadores planteando preguntas sobre si la empresa actúa de modo ético, sobre el fin último de la empresa y sus responsabilidades con respecto a sus empleados y sobre cuál sea la connotación social en la que deben basarse (Yarce, 2006).

En este punto radica la importancia de la presente investigación: la empresa es una estructura social bien delimitada conformada por personas las cuales persiguen el objetivo social de la misma empresa, pero que al mismo tiempo la empresa debe desarrollar su aportación para que al momento de alcanzar los objetivos empresariales, también esté cubriendo el alcance de los objetivos personales de los trabajadores.

En la actualidad el porcentaje de empleados leales a la organización ha disminuido considerablemente sin que los empleadores consideren este factor como algo negativo para esta, toda vez que se basan en la consideración de que cumpliendo los objetivos organizacionales por medio de la fuerza laboral, no existe trascendencia en la lealtad del trabajador. Este principio está basado en la ley de la oferta y demanda laboral por lo que al incrementarse la oferta de trabajadores (por sobrepoblación), las empresas tienen mayor mercado de donde reclutar y seleccionar a su capital humano. Sin embargo, debe tomarse conciencia también, del costo que implica no solo el reclutamiento y la selección; sino también de los costos de capacitación, desarrollo e integración a los equipos de trabajo (Osterman, 2009).

Actualmente se desconoce el grado de lealtad hacia la organización por parte de los colaboradores de la institución objeto de estudio por las características propias de su contratación y su desarrollo profesional. Además, la rotación de personal es inferior al $5 \%$ por ciclo semestral, los colaboradores no se involucran en su totalidad con la normatividad oficial de la organización incluyendo las actividades de capacitación, elaboración de materiales didácticos, seguimiento del programa académico al cual se encuentran adheridos y manifiestan desconocimiento o apatía ante nuevos proyectos, propios de cada coordinación académica. Carecen también por parte de la organización de un plan de desarrollo integral y plan de carrera para los colaboradores del instituto objeto de investigación en relación directa con la mejora del capital humano.

En la presente investigación se estableció como objetivo determinar los factores que están presentes en el fenómeno de la lealtad en una institución de educación superior de la ciudad de Celaya, así mismo identificar la relación de estos factores acerca de la lealtad de estos colaboradores.

\section{MARCo TEÓRICo}

En un primer estudio se señala que es ampliamente aceptado que la lealtad es un factor determinante en el éxito de relaciones de largo plazo en organizaciones de servicio, por lo cual la consolidación y la mejora de la lealtad a la organización se consideran como estratégica. En el ámbito educativo, la lealtad significa un profundo compromiso a matricularse en una institución educativa preferida de forma consistente en el futuro, recomendar el servicio a otros y animarlos a utilizarlo (Cavazos y Encinas, 2016).

En otro estudio se generó un modelo que permitió comprender cómo se forma la lealtad del consumidor en un ámbito de servicios, los resultados muestran un buen ajuste estadístico del modelo propuesto, así como la comprobación de las hipótesis formuladas, lo que muestra lo adecuado del modelo en la explicación de la 
lealtad del consumidor en ambientes de servicio. Por tanto, se muestra evidencia que la utilización de efectos mediadores es significativa y explica mejor las variables satisfacción y lealtad (Bustamante, 2015).

La corriente denominada concepto integrador de lealtad propone la lealtad como un constructo multidimensional definido, principalmente, por comportamientos y actitudes. En donde estudiaron específicamente la lealtad desde tres perspectivas: (a) la lealtad comportamental, asociada a los comportamientos repetitivos; (b) la lealtad afectiva, entendida como el vínculo emocional, y (c) la lealtad cognitiva, explicada por la atención y concentración que una persona presta a procesos. Estas tres dimensiones se denominan dimensión comportamental, dimensión cognitiva y dimensión (Duque y Ramírez, 2015).

Otro estudio efectuado por Ernesto Duarte Magaña, en el 2010 en Cetys Universidad, compara las relaciones entre la satisfacción en el trabajo, las actitudes de los empleados y la productividad, desarrollados en los Estados Unidos y Europa para construir un modelo regional, este trabajo de investigación tiene como base las teorías de satisfacción laboral fundadas en necesidades de suficiencia y de crecimiento. Las necesidades de crecimiento se estratifican en afiliación y autoridad. El análisis estratificado de las teorías de la satisfacción laboral comprendidas en este estudio, también incluye a la estructura dual del comportamiento con factores motivacionales e higiénicos, subdividiéndose estos últimos en existentes y relativos. Asimismo, se estudian las teorías de satisfacción basadas en procesos como la teoría de expectativas y el modelo de expectativa de recompensas intrínsecas y extrínsecas.

Para Edelberg (2011) hablar de lealtad suele darse con relación a temas importantes: la lealtad para con la familia, los amigos o la patria, por ejemplo. Lealtades que, invocadas, obligan a determinados comportamientos. En el seno de las empresas: se habla de la lealtad de los trabajadores para con la organización, la de la organización para con sus trabajadores y, en el área comercial, la de los clientes para con la marca. En los albores del siglo XXI, luego de épocas de reingeniería, outsourcing, cierres de plantas y pérdidas de fuentes de trabajo, la lealtad en la empresa se puede describir, según distintas opiniones, como un anacronismo, una ambigüedad o una contradicción. No obstante, surgen voces nostálgicas que piden el regreso de la lealtad al seno de las empresas e indican la conveniencia de volver a ocuparse del tema. Si así fuera ¿qué se entiende por lealtad? Su significado no surge de inmediato. Se lo intuye; pero no siempre se lo explica de idéntica forma. Unos dicen que la lealtad de los trabajadores consiste en el vínculo con los jefes y el cumplimiento de sus instrucciones; otros, en el vínculo con la organización, manifestado en no renunciar para ir a trabajar a la competencia. A la inversa, la lealtad de la empresa para con los trabajadores consiste, para unos, en pagar sueldos justos o superiores a los del mercado; para otros, en no llevar a cabo despidos o en ambas cosas. Las dificultades surgen del hecho de que la lealtad en la empresa suele ser explicada desde un punto de vista operativo como desde otro que incluye aspectos de ética empresarial. En el primer caso el énfasis se pone en el tema de la retención de los empleados.

Un estudio elaborado por Edelberg (2011) indicó que solo un 27 por ciento de los trabajadores de empresas manufactureras planeaban quedarse dos años en la empresa. La gente, especialmente los mejores, es más leal a sus carreras que a su empleador. Estos no son mutuamente excluyentes si la organización ayuda al empleado a progresar en su carrera. Si el empleado se va, no es que sea desleal. La lealtad no es para siempre. Para lograr un equilibrio entre carrera y lealtad a la empresa, se recomienda alinear el crecimiento en la primera con los objetivos de la segunda; el diseñar las tareas con variedad y autonomía; desarrollar relaciones con supervisores y colegas y hacer énfasis en el vínculo entre los valores de los empleados y la misión de la empresa. Según Lee (2006), la satisfacción de los empleados no conduce a su lealtad para con la empresa. Los beneficios que se les brinde puede satisfacerlos; pero la lealtad es otra cosa. Los empleados estarán insatisfechos a menos que sus esfuerzos conduzcan a la lealtad de los clientes. Para esto recomienda equipos de trabajo no muy grandes, donde existe una dinámica que facilita los vínculos entre sus miembros. La lealtad en la empresa desde un punto de vista ético, por su parte, es mencionada de la siguiente manera: ¿Lealtad es lo mismo que compromiso? Algunos autores usan estas palabras en forma intercambiable, pese a que representan conceptos distintos y por lo tanto crean confusión. Compromiso tiene una fuerte conexión con la rotación de los 
trabajadores; mientras que lealtad se relaciona con el comportamiento que se espera de un empleado con principios morales o la posibilidad de denunciar hechos que atenten contra un comportamiento ético. La lealtad tiene una base moral para Lee (2006).

En este orden de ideas Ewin, (1992) lo expresa así: "La lealtad es fundamentalmente un vínculo y una reacción emocional hacia sus objetos; nuestras acciones no demuestran lealtad si son el resultado de un razonamiento frío. La persona leal es aquella que mantiene el vínculo a través de tiempos malos” (p. 407). La lealtad es la inclinación a formar vínculos emocionales con grupos de personas; es la expresión básica de la naturaleza social. La lealtad debe controlarse. Porque puede conducir a problemas si es excesiva o si se es leal a partir de objetos o bases equivocadas. No obstante esto último, las empresas y sus gerentes buscan la lealtad tanto de sus clientes como de sus empleados. El objetivo es lograr mayores ganancias. Una perspectiva cínica afirma que es posible que un empleado leal, manipulado a través de su salario, haga más por la empresa que lo indicado por un buen razonamiento.

De acuerdo con Heward (2015), este señala que la lealtad demanda persuasión total y que se ha de estar completamente convencido respecto a cualquier cosa en la cual se esté involucrado.

\section{MÉTodo}

Se trata de una investigación cuantitativa, en donde el diseño de investigación que se utilizó es del tipo no experimental transeccional, siendo su alcance de tipo correlacional debido a que busca establecer las relaciones entre las variables independientes y la variable dependiente del fenómeno analizado a través de un análisis estadístico de los coeficientes de correlación determinados entre las variables de estudio, sin incluir un modelo de regresión lineal múltiple, pues al tratarse de un estudio de caso no se pretende establecer un modelo matemático que intente explicar el comportamiento del fenómeno de la lealtad. (Hernández, Fernández y Baptista, 2014).

Siendo las hipótesis de esta investigación las siguientes:

$\mathrm{Hi}_{1}$; La variable denominada Recursos está relacionada sobre la variable Lealtad.

$\mathrm{Hi}_{2}$; La variable denominada Trabajo en equipo está relacionada sobre la variable Lealtad.

$\mathrm{Hi}_{3}$; La variable denominada Jefe directo está relacionada sobre la variable Lealtad.

$\mathrm{Hi}_{4}$; La variable denominada Gestión de la alta dirección está relacionada sobre la variable Lealtad.

$\mathrm{Hi}_{5}$; La variable denominada Retribuciones y Compensaciones está relacionada sobre la variable Lealtad.

La generación de los constructos de cada una de las variables fueron generados a partir de la revisión de literatura ejecutada en esta investigación los cuales se presentan a continuación: La lealtad es un estado emocional placentero o positivo que resulta de la apreciación del trabajo o de la experiencia laboral. La lealtad está sujeta a la razón, aunque también constituye una relación de reciprocidad; la lealtad se une a la afirmación y estabilidad de los principios y valores a través del tiempo. El trabajador leal se manifiesta fiel a ellos y estos confieren a su conducta una seguridad y constancia que lo impulsa a afrontar tanto dificultades como contradicciones con gran determinación y fortaleza. Y es precisamente en las dificultades de la organización cuando se manifiesta de manera inequívoca la lealtad a la misma.

Recursos, cuando en una institución u organización los recursos materiales o de trabajo son escasos o limitados surge la insatisfacción del colaborador al no disponer de estos para el buen desarrollo de sus funciones. Son elementos funcionales y necesarios para el logro de los objetivos individuales y de la misma organización. En una institución educativa existen recursos necesarios para el buen desempeño docente y administrativo, que al no poseerlos puede dañar el desarrollo de las actividades reflejándose directamente en un ineficiente servicio al cliente externo.

Trabajo en equipo. Tal como los define Bateman (2004); el trabajo en equipo se organiza con grupos formales, constituidos por individuos interdependientes que son responsables del logro de una meta. La formación de profesionistas en una institución educativa requiere de colaboradores que estén dispuestos a 
trabajar en equipo interrelacionando los conocimientos, habilidades y actitudes de formación por ciclo y por ende durante el proceso de formación total. Es crear un proceso formativo integral para el bienestar del cliente externo.

Jefe directo. La subordinación es parte secuencial de la vida organizacional previendo el cumplimiento de metas comunes y de normas institucionales por lo que es importante generar un vínculo armonioso con las personas que lideran a otras personas. Es parte de la vida misma.

Gestión de alta dirección. La tarea típica de la alta dirección es la toma de decisiones, la cual debe estar en sintonía con la misión y visión organizacional y absolutamente bajo el conocimiento de la ejecución de actividades y funciones de la totalidad de integrantes de la institución educativa. La correcta dirección de una organización, en un mundo tan competitivo como el actual, va más allá de la mera trasmisión de órdenes y requiere capacidades para tomar las decisiones que logren los objetivos a largo plazo, para organizar los recursos de manera eficiente y que sean capaces de controlar los procesos educativos e introducir los cambios necesarios en un entorno cambiante. Pero el desconocimiento puede causar desmotivación en el equipo de trabajo. Caso contrario, puede producir una enorme satisfacción laboral.

Retribuciones y compensaciones. Ya se mencionó que los sistemas de compensación fueron creados para fomentar determinadas conductas en el ámbito laboral. En materia de compensación y recursos humanos existe la creencia, el estereotipo financiero conductista el cual no ha sido abiertamente cuestionado, acerca de que el dinero motiva a trabajar (Osterman, 2009). En definitiva en tiempos de crisis económicas, tal como la que se vive hoy en día se puede afirmar que efectivamente el dinero sí motiva a los colaboradores no solo a trabajar sino a ser más eficientes y productivos en su área laboral.

De lo anterior se establecieron las variables que a continuación se describen en la Variable dependiente: Y1 Lealtad, la cual es operacionalizada a través de diez ítems, los cuales son adaptados del instrumento aplicado por Flores (2009) el cual fue validado a través de la técnica de juicios de expertos, estos ítems son de naturaleza ordinal debido a que se utiliza una escala de tipo Likert. También se establecieron las Variables independientes: X1 Recursos, X2 Trabajo en equipo X3 Jefe directo, X4 Gestión de la alta dirección y X5 Retribuciones y compensaciones cada una de ellas también operacionalizada con 10 ítems de naturaleza ordinal con una escala de tipo Likert, dando en total 50 ítems, al igual que con la variable dependiente se adaptaron del instrumento aplicado por Flores (2009).

\section{DETERMINACIÓN DEL TAMAÑo DE MUESTRA Y TÉCNICA DE MUESTREO}

Para la determinación del tamaño de la muestra se utilizó el algoritmo para poblaciones finitas, debido a que la cantidad de sujetos de investigación que conforman la población es conocida, por lo tanto se usa la siguiente Fórmula 1:

$$
n=\frac{z^{2}(p \cdot \mathrm{q}) \mathrm{N}}{\mathrm{NE}^{2}+z^{2}(p \cdot \mathrm{q})}
$$

[Fórmula 1]

Donde:

$\mathrm{n}=$ Tamaño de muestra buscada

$\mathrm{p}=$ representa la proporción del nivel de Lealtad que está presente en la población, derivado de estudios previos, para este caso se considerará un valor de 0.9

$\mathrm{q}=(1-\mathrm{p})$

$\mathrm{Z}=$ Unidades de desviación estándar que en la curva normal estandarizada están asociadas a una probabilidad acumulada, correspondiente a un intervalo o nivel de confianza, que para el caso de este estudio se empleó del 95\%. Por lo tanto, el valor de $\mathrm{Z}$ es de (para $\alpha=0.05, \mathrm{z}=1.96$ ) 
$\mathrm{E}=$ Error estándar de estimación, que para este estudio de determinó del 3\%.

Nivel de confianza del 95\%

Sustituyendo los datos en la Fórmula 2 se obtiene el siguiente resultado

$$
n=\frac{1.96^{2}(0.9 \cdot 0.1)(95)}{(95)\left(0.03^{2}\right)+\left(1.96^{2}(0.9 \cdot 0.1)\right)}=76.16 \approx 76
$$

[Fórmula 2]

Derivado de lo anterior el tamaño de muestra establecido para este estudio es de 76 sujetos de investigación, los cuales fueron seleccionados de forma probabilística a través de número aleatorios que les fueron asignados a cada sujeto que conforma la población,

En relación con la técnica de muestreo, se empleó la técnica de entrevista dirigida empleando el instrumento diseñado para la medición de las variables de estudio, lo anterior con la finalidad de reducir el error debido al instrumento y además incrementar la confiabilidad de este.

\section{CONFIABILIDAD DEL INSTRUMENTO}

La confiabilidad del instrumento documental se determinó utilizando la prueba estadística de coeficiente de Alfa de Cronbach para los ítems cualitativos y el resultado obtenido fue de 0.92, lo cual es interpretado como una confiabilidad del instrumento alta. Es necesario comentar que el coeficiente de Alfa de Cronbach se determinó utilizando la Ecuación 1 con el apoyo del software estadístico SPSS.

$$
a=\left(\frac{\mathrm{K}}{\mathrm{K}-1}\right) \cdot\left(\begin{array}{r}
\sum_{\mathrm{l} \mathrm{V}_{i}}^{k} \\
1-\frac{\mathrm{V}_{\mathrm{T}}}{\mathrm{V}}
\end{array}\right)
$$

[Ecuación 1]

Donde:

$\alpha=$ Alfa de Cronbach

$\mathrm{K}=$ número de ítems

$\mathrm{Vi}=$ Varianza de cada ítem

$\mathrm{VT}=$ Varianza total

Además, se precisa que se ha utilizado la Teoría Clásica de Test debido a que el tamaño de la muestra es menor a 500, por lo cual no es posible utilizar la Teoría de Respuesta al ítem, en consecuencia no es posible determinar los parámetros de habilidad, dificultad y discriminación de cada ítem. Adicionalmente, se debe agregar que no fue posible ejecutar un análisis factorial confirmatorio, debido a que el comportamiento de los ítems es del tipo no normal y con ello no se cumple con una de las premisas necesarias para poder efectuar dicho análisis factorial.

\section{Resultados}

Para el análisis estadístico se utilizó el software Statistical Package for the Social Sciences (SPSS), en donde el primer paso fue determinar al comportamiento de las variables de estudio, a través una prueba de normalidad de Kolgomorov - Smirnov la cual pretende probar la hipótesis estadística nula de que el comportamiento de 
la variable es normal contra la hipótesis alterna que el comportamiento de la variable es no normal, la fórmula empleada para este cálculo se presente en la Ecuación 2.

$$
\mathrm{D}=\max \left\{\mathrm{D}^{+}, \mathrm{D}^{-}\right\}
$$

[Ecuación 2]

Donde:

$$
\begin{gathered}
\mathrm{D}^{+}=\max _{l \leq i \leq n}\left\{\frac{i}{n}-\mathrm{F}_{0}\left(x_{i}\right)\right\} \\
\mathrm{D}^{-}=\max _{l \leq i \leq n}\left\{\mathrm{~F}_{0}\left(x_{i}\right)-\frac{i-1}{n}\right\}
\end{gathered}
$$

$\mathrm{X}_{\mathrm{i}}$ es el i-ésimo valor observado en la muestra (cuyos valores se han ordenado previamente de menor a mayor).

$$
\mathrm{F}_{0}\left(x_{i}\right)
$$

es la probabilidad de observar valores menores o iguales que xi cuando $\mathrm{H}_{0}$ es cierta.

El valor de $\mathrm{D}$ se compara contra el valor de contraste $\mathrm{D}_{\alpha}$, el cual se establece en función de la distribución a probar, que para el caso de la distribución normal se utiliza la Ecuación 3.

$$
\mathrm{D}_{a}=\frac{\mathrm{C}_{a}}{k(n)}
$$

[Ecuación 3]

Donde:

$$
\mathrm{C}_{a}
$$

$=0.895$ para una distribución normal con una alfa de 0.05

$$
k(n)=\sqrt{n}-0.01+\frac{0.85}{\sqrt{n}}
$$

Finalmente, se transforman los valores D obtenidos a su valor de probabilidad asintótico para cada una de las variables de estudio, en donde la forma de probar la hipótesis nula es que el valor asintótico encontrado sea mayor a el nivel de alfa (0.05), en otras palabras, el comportamiento de las variables es normal si el valor asintótico encontrado es mayor a 0.05 . Para el caso de este estudio todos los valores asintóticos encontrados fueron menores a 0.05 , por lo tanto el comportamiento de las variables de este estudio es no normal, lo anterior resulta relevante debido a que ayuda a establecer el estadístico de prueba que se estableció para probar la hipótesis de investigación.

Coeficientes de correlación de Tau-b de Kendall, es una medida no paramétrica de la correlación entre dos variables no normales. Es muy similar a los coeficientes de correlación de Spearman, sin embargo el coeficiente de correlación de Tau-b Kendall representa una probabilidad, en otras palabras esta representa la diferencia entre la probabilidad de los datos observados en el mismo orden contra la probabilidad de los datos observados en diferente orden.

La Ecuación 4 muestra cómo determinar la probabilidad del coeficiente de correlación de Tau-b Kendall a través de la diferencia entre los pares concordantes y pares discordantes. 


$$
\tau=\frac{\mathrm{C}-\mathrm{D}}{\mathrm{C}+\mathrm{D}}=\frac{\mathrm{C}-\mathrm{D}}{\frac{n(n-1)}{2}}=\frac{\mathrm{C}-\mathrm{D}}{\left(\begin{array}{l}
n \\
2
\end{array}\right)}=\frac{\mathrm{C}-\mathrm{D}}{\frac{n !}{2 !(n-2) !}}
$$

[Ecuación 4]

Donde:

$\mathrm{C}=$ Pares concordantes

$\mathrm{D}=$ Pares Discordantes

Fue así que con ayuda del software SPSS se generó una Tabla 1 de correlaciones sobre las variables cualitativas ordinales no normales para identificar las relaciones de influencia de las variables aplicando el coeficiente de correlación de Tau-b de Kendall. Dicho coeficiente es una medida de asociación lineal que utiliza los rangos, números de orden, de cada grupo de sujetos y compara dichos rangos. El coeficiente de correlación de Tau-b de Kendall es exactamente el mismo que el coeficiente de correlación de Pearson calculado sobre el rango de observaciones, la diferencia radica en que el primero es usado cuando el comportamiento de las variables es no normal. El coeficiente de correlación de Tau-b de Kendall es recomendable utilizarlo cuando los datos provienen de variables con comportamiento no normal. (Fernández y Díaz, 2001).

TABLA 1

Coeficientes de correlación de Kendall

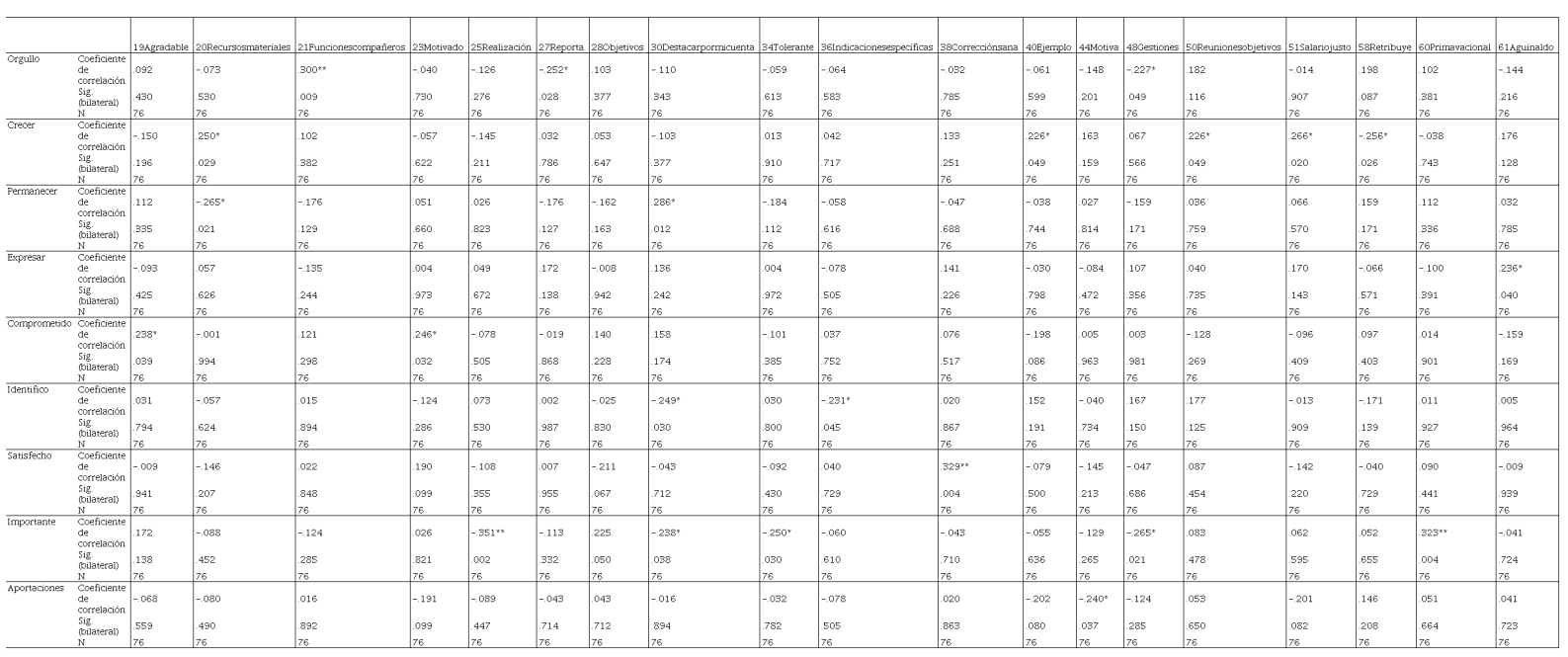

Fuente: Elaboración propia

Los resultados presentados en la Tabla 1 son únicamente aquellas correlaciones que resultaron significativas, no se incluye el resto de las correlaciones correspondientes a los ítems del resto de las variables, debido a que la dimensión de la Tabla 1 se haría demasiado grande, además de que los valores de significancia obtenidos son menores al valor crítico de 0.05 para el resto de los ítems no incluidos. Además para comprender mejor la Tabla 1 es pertinente señalar que la primer columna indica los ítems correspondientes a la variable dependiente Y1 Lealtad y los encabezados de las siguiente columnas corresponden a los ítems que resultaron significativos lo cuales corresponden solamente a las variables X2 Trabajo en equipo y X5 Retribuciones y compensaciones. Por lo cual, analizando los resultados de la Tabla 1 se observa que los valores obtenidos de significancia son mayores al valor crítico de 0.05 , lo cual indica que la correlación que existe entre los ítems de las variables X2 Trabajo en Equipo y X5 Retribuciones y Compensaciones con respecto a los ítems de la variable Y1 Lealtad son significativos. En otras palabras existe evidencia estadística que apoya las hipótesis de investigación $\mathrm{Hi}_{2}$; La variable denominada Trabajo en equipo influye sobre la variable Lealtad y $\mathrm{Hi}_{5}$; La variable denominada Retribuciones y compensaciones influye sobre la variable Lealtad. Además 
no se encontró evidencia estadística para aceptar las hipótesis de investigación $\mathrm{Hi}_{1}$; $\mathrm{La}_{\text {variable denominada }}$ Recursos influye sobre la variable Lealtad, $\mathrm{Hi}_{3}$; $\mathrm{La}$ variable denominada Jefe directo influye sobre la variable Lealtad, $\mathrm{Hi}_{4}$; La variable denominada Gestión de la alta dirección influye sobre la variable Lealtad, por lo tanto estas se rechazan.

\section{Conclusiones}

Se ha analizado en esta investigación el fenómeno de la lealtad en una institución educativa particular de educación superior en la ciudad de Celaya en el estado de Guanajuato, en donde a través de los resultados encontrados es posible afirmar que existe evidencia estadística que apoya la hipótesis de esta investigación $\mathrm{Hi}_{2}$; la variable denominada Trabajo en equipo influye sobre la variable Lealtad y $\mathrm{Hi}_{5}$; la variable denominada Retribuciones y compensaciones influye sobre la variable Lealtad. Lo anterior permite orientar los esfuerzos dentro de la Institución a mejorar y controlar las variables independientes encontradas para lograr incrementar la Lealtad, sin duda es de gran ayuda tener este conocimiento para la generación de planes de acción específicos. Sin embargo, se recomienda como trabajo futuro continuar con la replicación del método de investigación presentado y preferentemente ampliar el número de sujetos de investigación considerando más instituciones, con el objetivo de encontrar evidencia la cual confirme los resultados encontrados en esta investigación, además se generar un modelo de regresión lineal que intente explicar la relación de la Lealtad como variable dependiente y las variables independientes X2 Trabajo en equipo y X5 Retribuciones y compensaciones.

Por lo tanto, este estudio permite entender la importancia de la lealtad y el grado adquirido por los colaboradores, en la Institución a pesar de ser privada existe compromiso, pertinencia, al sentirse parte importante en el desempeño de sus funciones; en la presente investigación se pudo observar que los resultados dentro de la prueba de hipótesis se probó la importancia de que cada persona es capaz de proporcionar lealtad a la Organización, y se puede contrastar con el artículo denominado Lealtad, satisfacción y rendimiento académico en los estudiantes de la UASLP-UAMZM, que de manera representativa dentro de su investigación sus resultados muestran la existencia y tipo de lealtad de los alumnos (Gómez, Martínez, Recio y López, 2013). Por lo cual se puede considerar que el estudio está validado y probado, esto permite analizar que se trabaja al interior para lograr objetivos al exterior, es decir el compromiso probado de colaboradores permiten que los alumnos logren lealtad y se evite la deserción.

La presente investigación queda abierta para continuar en el sentido pertinente de ampliar y obtener resultados que permitan tener una reflexión en el sentido de la importancia de la lealtad, se recomienda a estudiantes, investigadores e investigadoras, docentes y personas en general que tengan la inquietud de conocer el proceso de lealtad de sus colaboradores.

\section{REFERENCIAS}

Bateman, T. S., Snell, S. (2004). Administración: una ventaja competitiva. Editorial Mc. Graw Hill: México.

Bustamante, J. C. (2015). Uso de variables mediadoras y moderadoras en la explicación de la lealtad del consumidor en ambientes de servicios. Estudios Gerenciales, 31(136), 299-309. https://doi.org/10.1016/j.estger.2015.05.002

Cavazos, J. y Encinas, F. C. (2016). Influencia del engagement académico en la lealtad de estudiantes de posgrado: un abordaje a través de un modelo de ecuaciones estructurales. Estudios Gerenciales, 32(140), 228-238. doi: https ://doi.org/10.1016/j.estger.2016.07.001

Duque, E. J.y Ramírez, P. J. (2015). Evolución conceptual y relación entre involucramiento y lealtad. Suma de Negocios, 5(12), 169-179. doi: https://doi.org/10.1016/s2215-910x(14)70039-4 
Edelberg, G. S. (2011). La lealtad en la empresa. INCAE Business School. Recuperado de https://nanopdf.com/down load/la-lealtad-en-la-empresa-guillermo-s-edelberg-dba_pdf

Ewin, R. E. (1992). Loyalty and Virtues. The Philosophical Quarterly, 42(169), 403-419.

Fernández, S. P. y Díaz S. (2001). Relación entre variables cuantitativas. Unidad de Epidemiología Clínica y Bioestadistica. España: Complexo Hospitalario Universitario de Coruña.

Flores, J. (2009). La lealtad en la cultura del trabajador mexicano de la ciudad de México. México: Textos Científicos.

Gómez, D., Martínez, E., Recio, R. y López, H. (2013). Lealtad, satisfacción y rendimiento académico en los estudiantes de la UASLP-UAMZM. Sophia, (9), 11-25.

Hernández, R., Fernández, C. y Baptista, P. (2014) Metodología de la Investigación. Editorial Mc. México: Graw Hill Heward, D. (2015). Lealtad y deslealtad. Recuperado de: https://books.google.com.mx/books?id=_itnDwAAQBAJ Lee, D. (2006). Convierta a su organización en un imán para atraer y retener talento. Blog AMA Global. Recuperado de https://bit.ly/2QCcCND

Osterman, P. (2009) Mandos Intermedios: Mejorando su Impacto en la Organización. México: Editorial PROFIT.

Yarce, J. (2006) El Poder de los Valores de las Organizaciones. México. Editorial Ruz.

\section{BY-NC-ND}

